\title{
Extensive Pneumomediastinum in COVID-19 Pneumonia
}

\author{
Adetiloye Oluwabusayo Adebola, MD*, Beketova Tatyana, MD, Williams Tabatha, NP and Agarwal \\ Sanket, MD
}

Department of Internal Medicine, Metropolitan Hospital Center, New York City Health + Hospitals Corporation, USA

*Corresponding author: Adetiloye Oluwabusayo Adebola, MD, Department of Internal Medicine, Metropolitan Hospital Center, New York City Health + Hospitals Corporation, $19011^{\text {st }}$ Avenue, Suite 705, New York, NY 10029, USA, Tel: +16465463690

\begin{abstract}
Pneumomediastinum, defined as the presence of air in the mediastinum often occurs due to trauma, mechanical ventilation or surgical procedure. It may also occur spontaneously due to predisposing lung diseases such as asthma and Chronic obstructive pulmonary airway disease (COPD). In this report, we present a case of a patient with COVID-19 pneumonia without any underlying lung conditions or usual risk factors for pneumomediastinum who developed extensive pneumomediastinum with pneumopericardium during the course of hospitalization.
\end{abstract}

\section{Keywords}

Spontaneous pneumomediastinum, COVID-19

\section{Introduction}

COVID-19 (Coronavirus disease 2019) is a novel disease whose pathophysiology, clinical course, management options and outcomes are still being elucidated. The disease was first reported in Wuhan China in December 2019 and was subsequently named "COVID-19" by the World Health Organization (WHO) on 11 February 2020 [1]. The clinical manifestations of COVID-19 pneumonia vary, ranging from mild flu-like symptoms to severe ARDS at the other end of the spectrum [2]. Other commonly reported complications are acute kidney injury, cardiac injury, liver dysfunction, thromboembolic disease and pneumothorax $[3,4]$. According to various literature, Spontaneous pneumomediastinum (SPM) is an infrequent, benign, and self-limiting condition with mortality generally attributable to underlying disease states [5-7]. Although typically seen in patients with underlying obstructive lung disease, it has also been described in patients with interstitial lung disease $[8,9]$. Only a few cases have been described in patients with COVID-19 infection $[10,11]$. This report highlights SPM as a potential complication of COVID-19 pneumonia.

\section{Case Report}

We present a 75-year-old male with hypertension and hyperlipidemia presented to the ED with a four-day history of shortness of breath, dry cough and myalgia. Vitals at the time of presentation were remarkable for tachycardia, tachypnea and hypoxia with oxygen saturation $90 \%$ on room air. Lung auscultation notable for bilateral rales. Chest radiograph revealed significant airspace disease throughout both lungs consistent with airspace disease. Initial labs revealed leukocytosis (white cell count $25 \times 103 / \mathrm{mcl}$ ), elevated inflammatory markers (ferritin $988 \mathrm{ng} / \mathrm{dl}, \mathrm{C}$-Reactive Protein $39 \mathrm{mg} /$ dl, Lactate Dehydrogenase 697 U/L, D-dimer 1244 ng/dl, fibrinogen $616 \mathrm{mg} / \mathrm{dl}$, Interleukin-6 $323 \mathrm{pg} / \mathrm{ml}$, procalcitonin $2.7 \mathrm{ng} / \mathrm{ml}$ ), lactic acidosis (Lactic acid $2.6 \mathrm{mmol} / \mathrm{L}$ ), elevated liver enzymes. COVID-19 PCR test through a nasal swab returned positive. Patient was admitted for severe pneumonia secondary to COVID-19 infection with superimposed bacterial pneumonia. He was commenced on supplemental oxygen via nasal cannula on medical floors, hydroxychloroquine, ceftriaxone, azithromycin, therapeutic doses of subcutaneous enoxaparin, vitamin $C$ and zinc supplement.

Over the next couple of days, his clinical condition kept worsening despite medical management with worsening of cough and shortness of breath being noted. His oxygen requirement also kept increasing to a 
point when he was being maintained on High-flow nasal cannula at $40 \mathrm{~L} / \mathrm{min}$ at $60 \%$ fraction of inspired oxygen. Inflammatory markers during this period trended up as well. After showing no improvement with hydroxychloroquine, ceftriaxone, azithromycin and enoxaparin, he was started on dexamethasone therapy with no response. Given inability to wean oxygen and worsening clinical condition, he received a dose of tocilizumab around day 15 after which his clinical condition started improving. His oxygen requirement also came down to $5 \mathrm{~L} / \mathrm{m}$ via nasal cannula. Soon he subjectively started feeling better in terms of the dyspnea but occasionally continued to complain of chest pain. The chest pain was diffuse and initially attributed to the underlying pleuritic pain from COVID-19 pneumonia.

However on hospital day 28 , he reported change in the character of the pain which was now much worse. He described central chest pain which was sharp, 8/10 in intensity, non-radiating. Auscultation findings of lungs were unchanged with equal air entry and clear auscultation bilaterally. His heart rate was noted to be high as well around 110 s to 120 s as compared to baseline of
80 s to 90 s, BP was noted to be in the normal range. Oxygen requirements were at $2 \mathrm{~L} / \mathrm{m}$ through a nasal cannula. EKG showed sinus tachycardia with normal sinus rhythm. A CT angiogram of chest was performed to rule out a pulmonary embolism. The scan showed no pulmonary embolism but surprisingly reported extensive pneumomediastinum and pneumopericardium extending to the soft tissues of the neck and downwards along the descending aorta into the abdomen. Interstitial and alveolar infiltrates, patchy consolidations predominantly in subpleural and peribronchial distribution were also observed (Figure 1). Following these significant findings, his oxygen supplementation device was escalated to non-rebreather face mask to provide maximal $\mathrm{FiO}_{2}$ in order to facilitate resorption of free air. Aggressive pain management, cough suppressants, stool softeners along with strict bed rest activity orders were initiated. His vitals were monitored closely and he continued having regular examinations and serial CT scans of chest to ensure early detection of tension pneumothorax and tension pneumomediastinum. With the above treatment, his chest pain kept improving and serial imaging of chest revealed improvement of the pneumomedi-

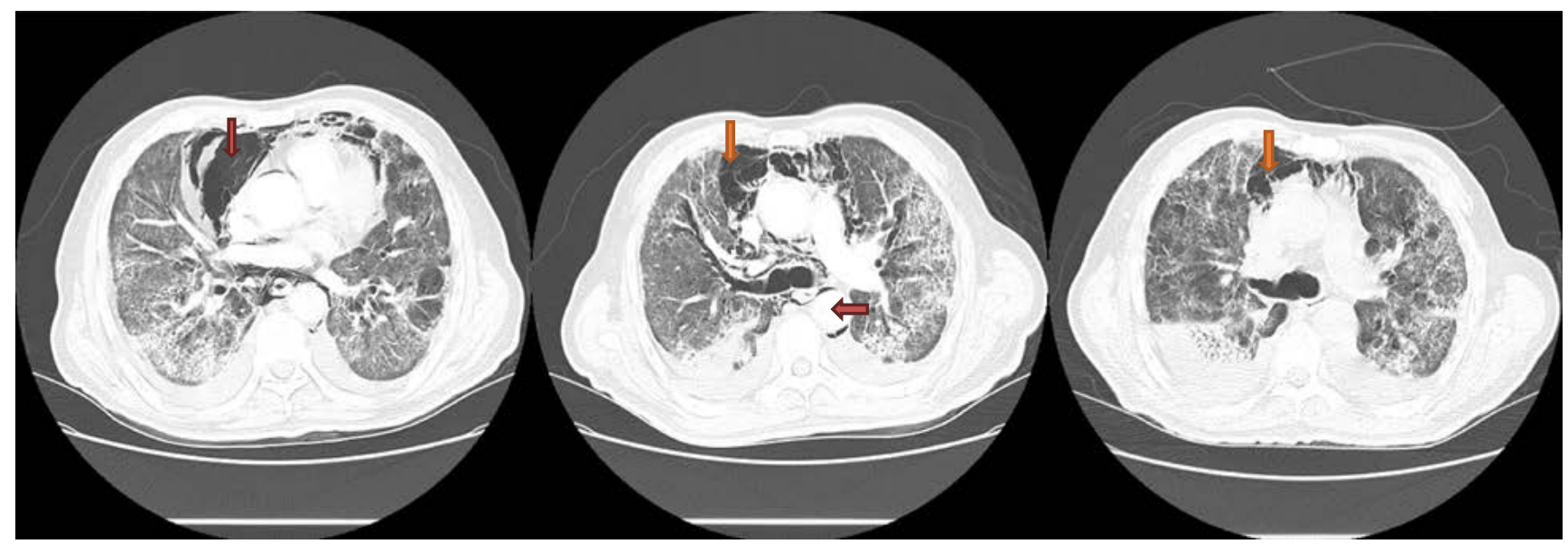

Figure 1: Extensive pneumomediastinum and pneumopericardium extending downwards along the descending aorta into the abdomen with interstitial and alveolar infiltrates. There are patchy consolidations predominantly in subpleural and peribronchial distribution (Day 28 of admission).

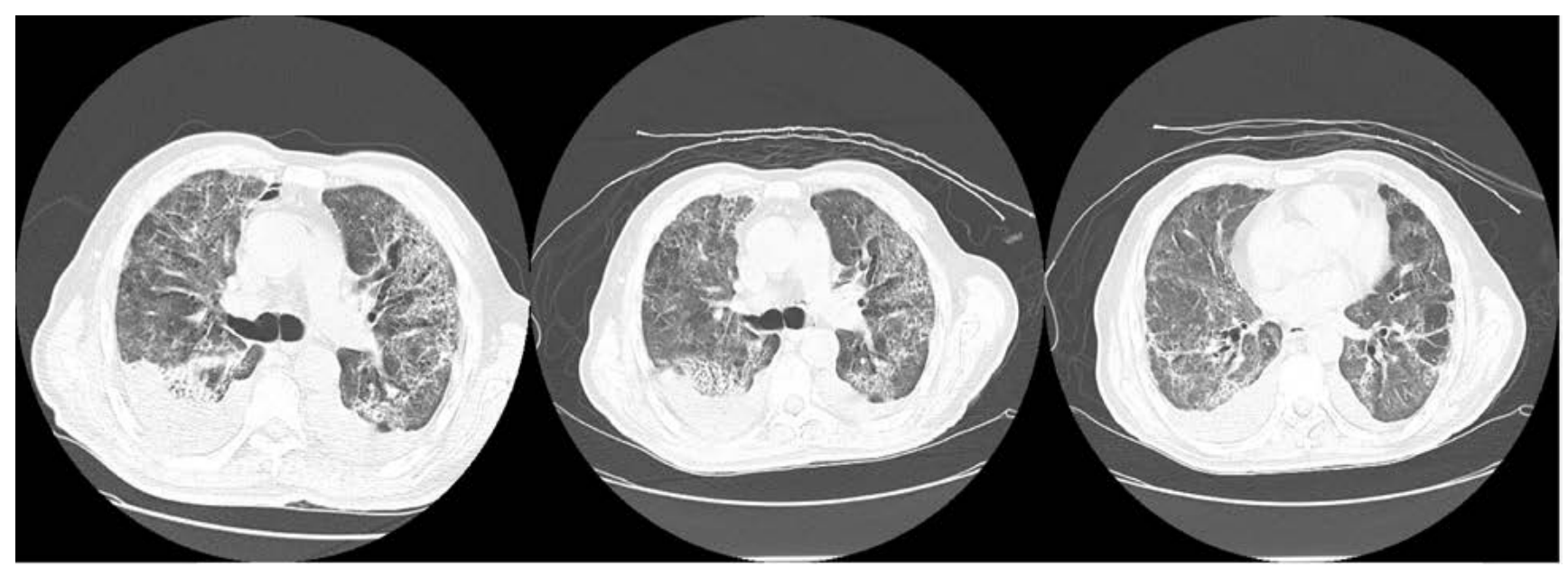

Figure 2: Pneumomediastinum and pneumopericardium almost completely resolved (Day 38 of admission). 
astinum and pneumopericardium. By hospital day 38 , his pneumomediastinum and pneumopericardium almost completely resolved (Figure 2). He was discharged home in a stable condition on day 54 .

\section{Discussion}

COVID-19 virus is capable of producing an excessive immune reaction in the host leading to extensive tissue damage [2] but the precise mechanism of SPM in COVID-19 is unknown. Based on existing literature, the likely sequence of events in the development of pneumomediastinum could involve rupture of damaged alveolar wall, air dissection along the bronchovascular sheath, and free air reaching the mediastinum [6]. Depending upon tissue planes involved, the air dissection could even extend to cause pneumopericardium, pneumothorax, pneumoperitoneum or subcutaneous emphysema [6]. Common triggering factors include events that elevate alveolar pressure such as mechanical ventilation, Valsalva maneuver or other causes such as central line placements [8]. Vigorous coughing, emesis or other maneuvers that increase the intrathoracic pressure are also other possible mechanisms [8]. We hypothesize that persistent cough was the likely etiology in this case as he never had any other risk factors or triggering events.

This case highlights the possibility of late onset severe life-threatening complication resulting from extensive COVID-19 pneumonia in an otherwise clinically improving patient needing physicians to be very vigilant. COVID-19 pneumonia is now known to commonly lead to pulmonary complications such as ARDS, acute respiratory failure, superimposed bacterial infection, pulmonary embolism and lung scarring but SPM is not a commonly seen phenomenon and therefore not usually suspected [12]. SPM is a life threatening condition that requires aggressive management. In this case, patient received supportive care and therapies targeted at reducing triggers such as excessive coughing along with high $\mathrm{FiO}_{2}$ supplemental oxygenation for better resorption of the pneumomediastinum. It is also crucial to distinguish pneumomediastinum from conditions with similar clinical findings that require immediate treatment, such as pulmonary embolism, cardiac tamponade, acute coronary syndrome, myopericarditis, aortic dissection and mediastinitis, some of which could occur as complications of COVID-19 infection or co-morbid conditions $[5,13]$. SPM can be managed by non-surgical approaches with good results as evidenced in this case, however, physicians should be vigilant to monitor for any complication such as progression to tension pneumothorax and tension pneumomediastinum which carry a high mortality [8].

\section{Conclusion}

We conclude that worsening chest pain, tachycardia, and oxygen desaturation in a patient with severe
COVID-19 pneumonia necessitates prompt imaging and close monitoring due to the possibility of spontaneous pneumomediastinum. With the staggering number of COVID-19 cases worldwide, awareness regarding SPM as its serious complication is necessary among clinicians.

\section{Declaration of Interest}

We declare no competing interests.

\section{References}

1. Wang $Y$, Wang $Y$, Chen $Y$, Qin $Q$ (2020) Unique epidemiological and clinical features of the emerging 2019 novel coronavirus pneumonia (COVID-19) implicate special control measures. J Med Virol 92: 568-576.

2. Cascella M, Rajnik M, Cuomo A, Dulebohn SC, Di Napoli $R$ (2021) Features, evaluation and treatment coronavirus (COVID-19). Stat Pearls Publishing

3. Yang X, Yu Y, Xu J, Shu H, Xia J, et al. (2020) Clinical course and outcomes of critically ill patients with SARSCoV-2 pneumonia in Wuhan, China: A single-centered, retrospective, observational study. Lancet Respir Med 8: 475-481.

4. Sakr Y, Giovini M, Leone M, Pizzilli G, Kortgen A, et al. (2020) Pulmonary embolism in patients with coronavirus disease-2019 (COVID-19) pneumonia: A narrative review. Ann Intensive Care 10: 124.

5. Langwieler TE, Steffani KD, Bogoevski DP, Mann O, Izbicki JR (2004) Spontaneous pneumomediastinum. Ann Thorac Surg 78: 711-713.

6. Carolan PL (2019) Pneumomediastinum. Medscape.

7. Zhou C, Gao C, Xie Y, Xu M (2020) COVID-19 with spontaneous pneumomediastinum. Lancet Infect Dis 20: 510.

8. Bejvan SM, Godwin JD (1996) Pneumomediastinum: Old signs and new signs. AJR Am J Roentgenol 166: 10411048.

9. Nadig N, Thomson C (2011) Pneumomediastinum in interstitial lung disease-A case report. Respir Med CME 4: 79-80.

10. Sun R, Liu H, Wang X (2020) Mediastinal emphysema, giant bulla, and pneumothorax developed during the course of COVID-19 pneumonia. Korean J Radiol 21: 541-544.

11. Wang J, Su X, Zhang T, Zheng C (2020) Spontaneous pneumomediastinum: A probable unusual complication of coronavirus disease 2019 (COVID-19) pneumonia. Korean J Radiol 21: 627-628.

12. Galiatsatos $P(2020)$ What coronavirus does to the lungs. Johns Hopkins Medicine.

13. Long B, Brady WJ, Koyfman A, Gottlieb M (2020) Cardiovascular complications in COVID-19. Am J Emerg Med 38: 1504-1507. 